\title{
204. Remarks on the Asymptotic Behavior of the Solutions of Certain Third Order Non-Autonomous Differential Equations
}

\author{
By Minoru Yamamoto \\ Osaka University
}

(Comm. by Kenjiro ShodA, M. J. A., Sept. 13, 1971)

1. Introduction. The differential equations considered here are of the form

$$
\begin{gathered}
\dddot{x}+\psi(t, x, \dot{x}, \ddot{x})+\phi(t, x, \dot{x})+c(t) f(x)=0 \\
\dddot{x}+\psi(t, x, \dot{x}, \ddot{x})+\phi(t, x, \dot{x})+c(t) f(x)=e(t)
\end{gathered}
$$

where $\psi, \phi, c, f$ and $e$ are real valued functions. The dots indicate differentiation with respect to $t$ and all solutions considered are assumed real.

In [3] Swick considered the behavior as $t \rightarrow \infty$ of solutions of the differential equations

$$
\begin{gathered}
\dddot{x}+a \ddot{x}+g(x) \dot{x}+h(x)=e(\mathrm{t}) \\
\dddot{x}+p(t) \ddot{x}+q(t) g(\dot{x})+h(x)=e(t)
\end{gathered}
$$

where $a$ is a positive constant. In [4] Swick also considered the asymptotic stability in the large, as $t \rightarrow \infty$, of the zero solution of the differential equations

$$
\begin{gathered}
\dddot{x}+p(t) \ddot{x}+q(t) g(\dot{x})+r(t) h(x)=0 \\
\dddot{x}+f(x, \dot{x}, t) \ddot{x}+q(t) g(\dot{x})+r(t) h(x)=0 .
\end{gathered}
$$

Recently, in [1] T. Hara obtained some conditions under which all solutions of the equations

$$
\begin{gathered}
\dddot{x}+a(t) \ddot{x}+b(t) \dot{x}+c(t) x=0 \\
\dddot{x}+a(t) f(x, \dot{x}) \ddot{x}+b(t) g(x, \dot{x}) \dot{x}+c(t) x=0
\end{gathered}
$$

tend to the zero solution as $t \rightarrow \infty$.

In [5], the author established conditions under which all solutions of the non-autonomous equation (1.1) tend to the zero solution as $t \rightarrow \infty$.

In this note we investigate the asymptotic behavior of the solutions of the equation (1.2), and the asymptotic stability in the large of the zero solution of the equation (1.1) under a condition slightly weaker than that obtained in [5].

Many results have been obtained on the asymptotic property of autonomous equations of third order and many of these results are summarized in [2].

2. Assumptions and Theorems. We shall state the assumptions on the functions $\psi, \phi, f, c$ and $e$ appeared in the equations (1.1) and (1.2). 
Assumptions.

( I ) $f(x)$ is a $C^{1}$-function in $R^{1}$, and $c(t)$ is a $C^{1}$-function in $I$ $=[0,+\infty)$.

(II) The function $\phi(t, x, y)$ is continuous in $I \times R^{2}$, and for the function $\phi(t, x, y)$ there exist functions $b(t), \phi_{0}(x, y)$ and $\phi_{1}(x, y)$ which satisfy the inequalities

$$
b(t) \phi_{0}(x, y) \leqq \phi(t, x, y) \leqq b(t) \phi_{1}(x, y)
$$

for all $(t, x, y) \in I \times R^{2}$. Moreover $b(t)$ is a $C^{1}$-function in $I$, and functions $\phi_{0}(x, y), \phi_{1}(x, y), \frac{\partial \phi_{0}}{\partial x}(x, y)$ and $\frac{\partial \phi_{1}}{\partial x}(x, y)$ are continuous in $R^{2}$.

(III) The function $\psi(t, x, y, z)$ is continuous in $I \times R^{3}$, and for the function $\psi(t, x, y, z)$ there exist functions $a(t), \psi_{0}(x, y)$ and $\psi_{1}(x, y)$ which satisfy the inequalities

$$
a(t) \psi_{0}(x, y) \cdot z \leqq \psi(t, x, y, z) \leqq a(t) \psi_{1}(x, y) \cdot z
$$

for all $(t, x, y, z) \in I \times R^{3}$. Further $a(t)$ is a $C^{1}$-function in $I$, and functions $\psi_{0}(x, y), \psi_{1}(x, y), \frac{\partial \psi_{0}}{\partial x}(x, y)$ and $\frac{\partial \psi_{1}}{\partial x}(x, y)$ are continuous in $R^{2}$.

Hereafter the following notations are used:

$$
\tilde{\phi}(x, y)=\frac{1}{2}\left\{\phi_{0}(x, y)+\phi_{1}(x, y)\right\}, \quad \tilde{\psi}(x, y)=\frac{1}{2}\left\{\psi_{0}(x, y)+\psi_{1}(x, y)\right\}
$$

Theorem 1. Suppose that the assumptions (I), (II) and (III), and that these functions satisfy the following conditions:

(1) $f(x) \cdot \operatorname{sgn} x>0, \quad f^{\prime}(x) \leqq f_{1} \quad\left(x \in R^{1}\right)$

( 2) $\quad F(x)=\int_{0}^{x} f(\xi) d \xi \rightarrow+\infty \quad(|x| \rightarrow \infty)$

(3) $\frac{\tilde{\phi}(x, y)}{y} \geqq \phi_{0}>0(y \neq 0), \tilde{\phi}_{x}(x, y) \leqq 0$ in $R^{2}, \phi(t, x, 0)=0$ in $I \times R$

(4) $\tilde{\psi}(x, y) \geqq \psi_{0}>0, \quad \psi_{x}(x, y) y \leqq 0 \quad$ in $R^{2}$

( 5) $\quad 0<c_{0} \leqq c(t) \leqq c_{1}, \quad 0<b_{0} \leqq b(t) \leqq b_{1}, \quad 0<a_{0} \leqq a(t) \leqq a_{1} \quad(t \in I)$

(6) $\sup _{y \neq 0} \frac{1}{y}\left\{\phi_{1}(x, y)-\phi_{0}(x, y)\right\}=p_{1}<+\infty$

$\sup _{y}\left\{\psi_{1}(x, y)-\psi_{0}(x, y)\right\}=q_{1}<+\infty$

( 7 ) $a_{0} b_{0} \phi_{0} \psi_{0}>c_{1} \cdot \max \left\{1, f_{1}\right\}$.

( 8 ) $4\left(\mu b_{0} \phi_{0}-c_{1} f_{1}\right) \cdot\left(a_{0} \psi_{0}-\mu\right) \geqq\left(\mu a_{1} q_{1}+b_{1} p_{1}\right)$

where $\mu$ is an arbitrarily fixed constant satisfying

$a_{0} \psi_{0}>\mu>\frac{c_{1}}{b_{0} \phi_{0}} \cdot \max \left(1, f_{1}\right)$

(9) $\sup _{t \in I}\left[\mu^{2}\left|\frac{a^{\prime}(t)}{a_{0}}\right|+\frac{1}{\lambda}\left\{\left|\frac{b^{\prime}(t)}{b_{0}}\right| \cdot c(t)-c^{\prime}(t)\right\}\right]<\mu b_{0} \phi_{0}-c_{1} f_{1}$ 
where $\lambda$ is an arbitrarily fixed constant which satisfies

$$
\mu>\lambda f_{1}>\frac{c_{1} f_{1}}{b_{0} \phi_{0}}
$$

$$
\begin{aligned}
& \int_{0}^{\infty}\left|a^{\prime}(t)\right| d t<+\infty, \int_{0}^{\infty}\left|b^{\prime}(t)\right| d t<+\infty, \int_{0}^{\infty}\left|c^{\prime}(t)\right| d t<+\infty \\
& c^{\prime}(t) \rightarrow 0 \text { as } t \rightarrow \infty \\
& \int_{0}^{\infty}|e(t)| d t<+\infty .
\end{aligned}
$$

Then every solution of (1.2) satisfies

$$
x(t) \rightarrow 0, \quad \dot{x}(t) \rightarrow 0, \quad \ddot{x}(t) \rightarrow 0 \quad(t \rightarrow \infty) .
$$

Theorem 2. Suppose that the assumptions (I), (II) and (III), and the conditions (1)-(11) of Theorem 1, then the zero solution of the equation (1.1) is asymptotically stable in the large as $t \rightarrow \infty$.

Remark. If the equation (1.1) reduces to an autonomous equation, i.e. the functions $\phi(t, x, y)$ and $\psi(t, x, y, z)$ are independent of $t$, and $c(t)$ is a constant, the conditions (6), (8), (9), (10) and (11) are always satisfied, and our result contains that obtained by Goldwyn-Narendra [2] and the like.

When the equation (1.1) reduces to a linear differential equation with constant coefficients, the condition described in Theorem 2 reduces to Routh-Hurwitz's condition.

3. Proof of Theorems. Before proving Theorems we state a well-known lemma obtained by T. Yoshizawa [6].

Auxiliary lemma. Consider the system $\dot{X}=F(t, X)+G(t, X)$, where $\int_{0}^{t}\|G(s, X)\| d s$ is bounded for all t whenever $X\left(x_{1}, \cdots, x_{n}\right)$ belongs to any compact subset of $R^{n}$. Suppose that there exists a non-negative Liapunov function $V(t, X)$ on $I \times Q, Q \subset R^{n}$, such that with respect to this system $\dot{V}(t, X) \leqq-W(X)$, where $W(X)$ is positive definite with respect to a closed set $\Omega$ in the space $Q$. Moreover, suppose $F(t, X)$ is bounded for all $t$ when $X$ belongs to an arbitrary compact set in $Q$ and that $F(t, X)$ satisfies the following conditions with respect to $\Omega$ :

(a) $F(t, X)$ tends to a function $H(X)$ for $X \in \Omega$ as $t \rightarrow \infty$, and on any compact set in $\Omega$ this convergence is uniform.

(b) Corresponding to each $\varepsilon>0$ and each $Y \in \Omega$, there exist $\delta(\varepsilon, Y)$ and $T(\varepsilon, Y)$ such that if $\|X-Y\|<\delta(\varepsilon, Y)$ and $t \geqq T(\varepsilon, Y)$, we have $\|F(t, X)-F(t, Y)\|<\varepsilon$.

Then every bounded solution of $\dot{X}=F(t, X)+G(t, X)$ approaches the largest semi-invariant set of the system $\dot{X}=H(X)$ contained in $\Omega$ as $t \rightarrow \infty$.

Proof of Theorem 1. First we note that the equation (1.2) is equivalent to the following system of differential equations :

$$
\dot{x}=y, \quad \dot{y}=z, \quad \dot{z}=e(t)-c(t) f(x)-\phi(t, x, y)-\psi(t, x, y, z) .
$$


A Liapunov function satisfying the conditions assumed in the auxiliary lemma will be given as follows:

$$
U(t, x, y, z)=e^{-\int_{0}^{t}[\rho(s)+|e(s)|] d s} \cdot V(t, x, y, z)
$$

where $\rho(s)=\left|\frac{a^{\prime}(s)}{a_{0}}\right|+\left|\frac{b^{\prime}(s)}{b_{0}}\right|+\left|\frac{c^{\prime}(s)}{c_{0}}\right|$ and

$2 \cdot V(t, x, y, z)$

$$
\begin{aligned}
= & 2 \mu \cdot c(t) \int_{0}^{x} f(\xi) d \xi+2 c(t) f(x) y+2 b(t) \int_{0}^{y} \tilde{\phi}(x, \eta) d \eta \\
& +2 \mu \cdot a(t) \int_{0}^{x} \tilde{\psi}(x, \eta) \eta d \eta+2 \mu y z+z^{2}+k \\
= & 2 c(t) \int_{0}^{x}\left\{\mu-\lambda f^{\prime}(\xi)\right\} f(\xi) d \xi+2 c(t)\left\{\sqrt{\lambda} f(x)+\frac{y}{\sqrt{\lambda}}\right\}^{2}+(\mu y+z)^{2} \\
& +\frac{2}{\lambda} \int_{0}^{y}\{\lambda b(t) \tilde{\phi}(x, \eta)-c(t) \eta\} d \eta+2 \mu \cdot \int_{0}^{y}\{a(t) \tilde{\psi}(x, \eta)-\mu\} \eta d \eta+k .
\end{aligned}
$$

( $k$ is a non-negative constant to be determined below.)

Then we easily have

$2 V(t, x, y, z)$

$$
\geqq 2 c_{0}\left(\mu-\lambda f_{1}\right) \cdot F(x)+\delta \cdot z^{2}+\left\{\frac{1}{\lambda}\left(\lambda b_{0} \phi_{0}-c_{1}\right)+\mu\left(a_{0} \psi_{0}-\frac{\mu}{1-\delta}\right)\right\} y^{2}+k,
$$

where $\delta$ is a suitable positive number satisfying $1>\delta>0$ and $a_{0} \psi_{0}(1-\delta)>\mu$. Therefore we can find a continuous function $w_{1}(r)$ with the properties $w_{1}(r) \geqq 0$ for $r \geqq 0, w_{1}(r) \rightarrow \infty$ as $r \rightarrow \infty$ and $w_{1}(\|X\|)$ $\leqq U(t, x, y, z)$ for all $X \in R^{3}$ and $t \in I$, where $\|X\|=\sqrt{x^{2}+y^{2}+z^{2}}$. The existence of a continuous function $w_{2}(r)$ which satisfies the inequality $U(t, x, y, z) \leqq w_{2}(\|X\|)$ for all $X \in R^{3}$ and $t \in I$, is easily verified.

Let $(x(t), y(t), z(t))$ be any solution of (3.1); then along this solution

$$
\begin{aligned}
2 \dot{V}_{(3.1)} & (t, x, y, z) \\
= & 2 \frac{d}{d t} V(x(t), y(t), z(t)) \\
= & 2 \mu c^{\prime}(t) \int_{0}^{x}\left\{1-\frac{\lambda}{\mu} f^{\prime}(\xi)\right\} f(\xi) d \xi+c^{\prime}(t)\left\{\sqrt{\lambda} f(x)+\frac{y}{\sqrt{\lambda}}\right\}^{2} \\
& -2\left\{\mu \phi(t, x, y)-c(t) f^{\prime}(x) y\right\} y-2\{\psi(t, x, y, z)-\mu z\} z \\
& +2 \int_{0}^{y}\left\{b^{\prime}(t) \tilde{\phi}(x, \eta)-\frac{c^{\prime}(t)}{\lambda} \eta\right\} d \eta+2 \mu a^{\prime}(t) \int_{0}^{y} \tilde{\psi}(x, \eta) \eta d \eta \\
& +2 b(t) y \cdot \int_{0}^{y} \tilde{\phi}_{x}(x, \eta) d \eta+2 \mu a(t) y \int_{0}^{y} \tilde{\psi}_{x}(x, \eta) \eta d \eta \\
& +2\{b(t) \tilde{\phi}(x, y)-\phi(t, x, y)\} z+2 \mu\{a(t) \tilde{\psi}(x, y) z-\psi(t, x, y, z)\} y \\
& -2(\mu y+z) e(t)
\end{aligned}
$$

for $t \geqq 0$.

Therefore along the solution $(x(t), y(t), z(t))$ of (3.1) 


$$
\begin{aligned}
& 2 e^{\int_{0}^{t}[\rho(s)+|e(s)|\} d s} \cdot \dot{U}_{(3.1)}(t, x, y, z) \\
& =2 \dot{V}(t, x, y, z)-2\{\rho(t)+|e(t)|\} V(t, x, y, z) \\
& \leqq-2\left(\mu b_{0} \phi_{0}-c_{1} f_{1}\right) y^{2}-2\left(a_{0} \psi_{0}-\mu\right) z^{2}-2\left\{c(t) \rho(t)-c^{\prime}(t)\right\} \\
& \cdot \int_{0}^{x}\left\{\mu-\lambda f^{\prime}(\xi)\right\} f(\xi) d \xi-2\left\{\left|\frac{b^{\prime}(t)}{b_{0}}\right| \cdot b(t)-b^{\prime}(t)\right\} \cdot \int_{0}^{y} \tilde{\phi}(x, \eta) d \eta \\
& +\frac{1}{\lambda}\left\{\left|\frac{b^{\prime}(t)}{b_{0}}\right| c(t)-c^{\prime}(t)\right\} y^{2} \\
& -\frac{2}{\lambda}\left\{\left|\frac{\alpha^{\prime}(t)}{a_{0}}\right|+\left|\frac{c^{\prime}(t)}{c_{0}}\right|\right\} \cdot \int_{0}^{y}\{\lambda b(t) \tilde{\phi}(x, \eta)-c(t) \eta\} d \eta \\
& -2 \mu\left\{\left|\frac{b^{\prime}(t)}{b_{0}}\right|+\left|\frac{c^{\prime}(t)}{c_{0}}\right|\right\} \cdot \int_{0}^{y}\{a(t) \tilde{\psi}(x, \eta)-\mu\} \eta d \eta \\
& +2 \mu\left\{\left|\frac{a^{\prime}(t)}{a_{0}}\right| a(t)-a^{\prime}(t)\right\} \cdot \int_{0}^{y} \tilde{\psi}(x, \eta) \eta d \eta+\mu^{2}\left|\frac{a^{\prime}(t)}{a_{0}}\right| y^{2} \\
& -\left\{\rho(t) c(t)-c^{\prime}(t)\right\}\left\{\sqrt{\lambda} f(x)+\frac{y}{\sqrt{\lambda}}\right\}^{2}-\rho(t)(\mu y+z)^{2} \\
& -\rho(t) k-2 b(t) y \int_{0}^{y} \tilde{\phi}_{x}(x, \eta) d \eta+2 \mu a(t) y \int_{0}^{y} \tilde{\psi}_{x}(x, \eta) \eta d \eta \\
& +2\{b(t) \tilde{\phi}(x, y)-\phi(t, x, y)\} z+2 \mu\{a(t) \tilde{\psi}(x, y) z-\psi(t, x, y, z)\} \\
& -|e(t)|\left\{2(\mu y+z)^{2}-2|\mu y+z|+k\right\} \\
& \leqq\left[\mu^{2}\left|\frac{a^{\prime}(t)}{a_{0}}\right|+\frac{1}{\lambda}\left\{\left|\frac{b^{\prime}(t)}{b_{0}}\right| c(t)-c^{\prime}(t)\right\}-\left(\mu b_{0} \phi_{0}-c_{1} f_{1}\right)\right] y^{2}-\left(a_{0} \psi_{0}-\mu\right) z^{2} \\
& -\left\{\left(\mu b_{0} \phi_{0}-c_{1} f_{1}\right) y^{2}-\left(\mu a_{1} q_{1}+b_{1} p_{1}\right)|y z|+\left(a_{0} \psi_{0}-\mu\right) z^{2}\right\},
\end{aligned}
$$

when we set $k=1 / 2$, and thus we have,

$$
\begin{aligned}
& 2 e^{\int_{0}^{t}[\rho(s)+|e(s)|] d s} \dot{U}_{(3.1)}(t, x, y, z) \\
& \leqq-\left[\mu^{2}\left|\frac{a^{\prime}(t)}{a_{0}}\right|+\frac{1}{\lambda}\left\{\left|\frac{b^{\prime}(t)}{b_{0}}\right| c(t)-c^{\prime}(t)\right\}-\left(\mu b_{0} \phi_{0}-c_{1} f_{1}\right)\right] y^{2} \\
&-\left(a_{0} \psi_{0}-\mu\right) z^{2}=w_{3}(y, z)
\end{aligned}
$$

in view of conditions (1)-(13), $(k=1 / 2)$.

As a result of (3.7) and the existence of the functions $w_{1}(\|X\|)$ and $w_{2}(\|X\|)$ we note that all solutions of (3.1) are bounded. The auxiliary lemma will be used to complete the proof.

In system (3.1) we set

$$
F(t, X)=\left(\begin{array}{c}
y \\
z \\
-c(t) f(x)-\phi(t, x, y)-\psi(t, x, y, z)
\end{array}\right), \quad G(t, X)=\left(\begin{array}{c}
0 \\
0 \\
e(t)
\end{array}\right)
$$

$F(t, X)$ is bounded for all $t$ when $X$ belongs to an arbitrary compact set in $R^{3}$.

Since the function $w_{3}(y, z)$ is positive definite with respect to the closed set $\Omega=\left\{(x, y, z) \in R^{3} \mid y=0, z=0\right\}$, it follows that on $\Omega$ 
$F(t, x)=\left(\begin{array}{c}0 \\ 0 \\ -c(t) f(x)\end{array}\right)$, and since $c(t) \rightarrow c_{\infty}, F(t, X) \rightarrow H(X)=\left(\begin{array}{c}0 \\ 0 \\ -c_{\infty} f(x)\end{array}\right)$ as $t \rightarrow \infty$. It is easy to show that the conditions (a) and (b) of auxiliary lemma are satisfied, and since every solution of (3.1) is bounded it follows from the auxiliary lemma that every solution of (3.1) approaches the largest semi-invariant set of $\dot{X}=H(X)$ contained in $\Omega$ as $t \rightarrow \infty$.

$\dot{X}=H(X)$ is the system

$$
\dot{x}=0, \quad \dot{y}=0, \quad \dot{z}=-c_{\infty} f(x)
$$

which has solutions $x=\gamma_{1}, y=\gamma_{2}, z=-c_{\infty} f\left(\gamma_{1}\right)\left(t-t_{0}\right)+\gamma_{3}$. To remain in $\Omega, \gamma_{2}=0, \gamma_{3}=0, f\left(\gamma_{1}\right)=0$ and thus $\gamma_{1}=\gamma_{2}=\gamma_{3}=0$. Therefore the only solution of $\dot{X}=H(X)$ remained in $\Omega$ is $X=0$, that is, the largest semiinvariant set of the system $\dot{X}=H(X)$ is $\Omega$ coincides with the point $(0,0,0)$. Thus we have the conclusion.

Q.E.D.

Proof of Theorem 2. The main tool in the proof of Theorem 2 will be the same Liapunov function as used in the proof of Theorem 1 , where in this case $k$ is equal to zero.

Remark. It should be noted that Theorem 1 remains true if we replace the equation (1.2) by the equation

$(1.2)^{\prime} \quad \ddot{x}+\psi(t, x, \dot{x}, \ddot{x})+\phi(t, x, \dot{x})+c(t) f(x)=e(t, x, \dot{x}, \ddot{x})$

where $e(t, x, y, z)$ is continuous in $I \times R^{3}$, and the condition (12) of Theorem 1 by

$(12)^{\prime}$

$$
\begin{aligned}
& |e(t, x, y, z)| \leqq \widetilde{e}(t) \quad \text { for } \forall(x, y, z) \in R^{3} \\
& \text { where } \widetilde{e}(t) \text { is a continuous function in I and satisfies } \\
& \int_{0}^{\infty} \widetilde{e}(t) d t<+\infty .
\end{aligned}
$$

In this case the proof is analogous to that of Theorem 1 if we take $\widetilde{e}(s)$ instead of $e(s)$ in the Liapunov function $U(t, x, y, z)$.

\section{References}

[1] T. Hara: On the stability of solutions of certain third order ordinary differential equations. Proc. Japan Acad., 47, 897-902 (1971).

[2] R. Reissig, G. Sansone und R. Conti: Nichtlineare Differentialgleichungen Höherer Ordnung. Roma (1969).

[3] K. E. Swick: Asymptotic behavior of the solutions of certain third order differential equations. SIAM. J. Appl. Math., 19, 96-102 (1970).

[4] - : On the boundedness and the stability of solutions of some nonautonomous differential equations of the third order. J. London Math. Soc., 44, 347-359 (1969).

[5] M. Yamamoto: On the stability of solutions of some non-autonomous differential equations of the third order. Proc. Japan Acad., 47, 909-914 (1971).

[6] T. Yoshizawa: Stability Theory by Liapunov's Second Method. The Math. Soc. of Japan (1966). 